\title{
LA HERMENÉUTICA DEL AUTOMÓVIL: UTOPÍA, (DES)MEMORIA Y METÁFORA
}

\author{
José-Luis ANTA FÉLEZ \\ Universidad de Jaén \\ Área de Antropología Social
}

http://dx.doi.org/10.5209/rev_NOMA.2013.v40.n4.48332

Resumen: A través de los correos electrónicos de un profesor de universidad vamos observando a lo largo de dos semanas cómo se construye, investiga y se polemiza sobre los automóviles como objeto privilegiado de la sociedad industrial y del mercado occidental. El automóvil por su propia dimensión de individualidad, pero también de objeto de consumo puede ser leído en tanto que es usado; se podría decir que el coche es el objeto social que representa lo individual, en cuanto que forma de la disciplina; a la vez que es el símbolo, también, de esa enorme maquinaria que es el poder. En última instancia, es un objeto que muestra las tensiones entre lo individual y lo social. De la misma manera, en este trabajo somos testigos de algunos entrecruzamientos en la vida de un profesor universitario que, generalmente, ni es lineal, ni mucho menos centrada únicamente en la academia, la docencia o la investigación, sino que tiene mucho de miserable, oportunista y de control de la vida social.

Palabras clave: Automóviles. Objetos. Capitalismo. Comunicación. Investigación.

\section{CAR'S HERMENEUTIC: UTOPIA, (DIS)MEMORY AND METAPHOR}

Summary: Through emails from a university professor we watching over two weeks is built, researched and polemic on cars as privileged object of industrial society and the western market. The car on its own dimension of individuality, but also consumer object can be read in that it is used, one could say that the car is the social object that represents the individual, in that form of discipline while which is the symbol, too, of that huge machine that is power. Ultimately, it is an object that reveals the tensions between the individual and the social. Similarly, in this work we witness some crossovers in the life of a university professor who, generally, nor is linear, far from focusing solely on academia, teaching or research, but it has a lot of miserable, opportunistic and control of the social life.

Keywords: Cars. Objects. Capitalism. Communication. Research. 
De: "PEÑALANDAJUANMANUEL" <jmpenal@autlook.com>

Asunto: Proyecto tesis

Fecha: 15 de abril de 2013 10:15:20 GMT+02:00

Para: José Luis Castillo <jlcastillo@ujaen.es>

Le adjunto la memoria de la tesis para su aprobación, recordándole la premura en los trámites, ya que las becas salen en Junio. En cualquier caso le quiero ir a ver una tarde de estas, me he pasado por su despacho varias veces pero nunca le encuentro. Saludos, Juan Manuel

\section{TITULO:LA HERMENÉUTICA DEL AUTOMÓVIL: UTOPÍA, (DES)MEMORIA Y METÁFORA}

\section{RESUMEN}

Entre todos los objetos de consumo que occidente ha producido, desde principios del siglo XX, el automóvil es, sin duda, uno de los más interesantes. Este concentra aplicaciones tecnológicas, estéticas, ideológicas e industriales que son difícilmente de encontrar en tal cantidad y calidad en otros productos. El automóvil ha conformado, además, en gran medida el cómo construimos la arquitectura, el urbanismo e, incluso, las relaciones sociales entre grupos, clases y sociedades. El automóvil, en cuanto objeto de consumo, sirve como evaluador del estado económico y social de las naciones y concentra de una manera esencial lo que los individuos piensan de sí mismos y cómo se muestran ante el ejercicio social. El automóvil como objeto de consumo es, sin duda, uno de los concentradores culturales privilegiados para entender, explicar y mostrar las sociedades industrializadas, e incluso, aún así, otras muchas que mantienen otros modelos culturales. Podría decirse que, desde un punto de vista de la sociología de los individuos, la historia del automóvil es la historia contemporánea de los mundos capitalistas y, mucho más, de los postcapitalistas.

Pero esta misma amplitud, este nivel de concentrar tantísimos elementos, lo hace especialmente complejo de observar y, mucho más, de interpretar. La industria del automóvil se mueve en parámetros socio-económicos de tal magnitud que lo hace inabordable, incluso, desde los análisis más micro y optimistas. En el mismo sentido el proceso estético, ideológico y de representación tiene tantas matizaciones sobre un mismo objeto que cualquier acercamiento no es sólo limitado y provisional, sino imperfecto y quizás muy poco realista. A todo ello se une, además, que la metodología para mirar es tan movedizacomo el objeto a mirar. Además, el automóvil es un producto estándar: la diferenciación y la repetición se dan en múltiples niveles y la frontera entre ambos es tan difusa como, en otros momentos, espesa. Como objeto de consumo éste tiene una vida social y simbólica que delimita espacios y tiempos, no siempre universales, y que tienen que ver con miradas específicas: puede que el automóvil esté fabricado en Japón o en Alemania, pero es simbolizado vitalmente allí donde se estaciona, conduce y muestra. Supera con mucho las barreras de lo transnacional, para hacerse, siempre, un elemento cotidiano y local. Igual ocurre cuando se le aplica la idea de gusto, que está traspasada por elementos de necesidad y deseo, donde la gran cantidad de marcas, modelos, terminaciones y colores lo hacen una pieza clave de la toma de decisiones y, aunque sea posible hacer divisiones de grupos y clases sociales por medio de las marcas y los modelos, el objeto tiende a revolverse sobre sí mismo -dejando perplejo no sólo a mi, sino, incluso, a los departamentos de marketing-, para tomar dimensiones simbólicas más allá del grupo social al que hipotéticamente se adscribe.

\section{PROPOSITO:}

Evidentemente el automóvil es un objeto de consumo que se mueve en los parámetros de la vida social, el mercado y la economía simbólica. Como cualquier otro objeto de consumo tiene unas características generales: es repetitivo, comerciable, finito, anunciado, funcional y está en relación con otros productos-objeto y servicios einstituciones; y otras específicas: es transformable, mitificado, representativo y asumido ideológicamente. Si contamos, además, que el automóvil es el segundo esfuerzo económico, tras la adquisición de una vivienda, del núcleo social de los mundos capitalistas y que sumado a lo largo de la vida de un individuo el principal gasto es de suponer que no todo es explicable ni como necesidad, ni como gusto, ni, mucho menos, como elemento propuesto funcionalmente. Lo que lejos de facilitar las cosas al científico social se las complica enormemente. Además, el esfuerzo industrial que rodea al 
automóvil es igualmente complejo y poderoso: desde que un automóvil es sólo una idea en los departamentos de diseño y marketing hasta que está en las manos del consumidor final pasan demasiadas cosas, casi ninguna lineal, para que se puedan simplificar hasta el nivel de su comprensión general y, mucho menos, su simplificación. Y si la estética del automóvil podría ser un ejemplo de los gustos del occidente capitalista, no es menos cierto que lo es también de sus aspiraciones, miedos y grandes valores, e, incluso, de sus avances técnicos, mecánicos y de estilo de hacer empresas y sociedades de forma global.

Lejos de ser una anécdota el que muchos estados, sobre todo asiáticos, impongan restricciones a la compra de un automóvil, en todos los Estados el nivel de fiscalidad, normatividad y legalidad, tanto en el cómo y el porqué del objeto, como en su uso y disfrute, lo hacen que tenga miradas de una cierta centralidad. A lo que se le tienen que sumar las no pocas industrias y empresas de servicio que se relacionan con el objeto coche de forma directa (seguros, talleres, repuestos...), indirecta (constructoras de carreteras, señales, combustibles, transportes...) y colateral (carreras de coches en diferentes modalidades, coleccionismo, catálogos, publicaciones...). De esta manera podemos decir que el automóvil no es sólo un concentrador cultural sino que también una pieza clave del mundo occidental $y$, consiguientemente, uno de los elementos más característicos para explicarlo, entenderlo e interpretarlo.

Si me he ido a un lugar tan ajeno como los automóviles para hacer ciencias sociales sólo puede ser entendido porque he buscado dentro de mi algo que me fuera tan incómodo, como extraño. Lo paradójico es que vivir en una sociedad sobre-informada nos hace que nada nos sea desconocido y, consecuentemente, parece como si las reacciones ante los hechos sólo fueran a nivel epidérmico. De hecho, el regreso a una antropología que se pregunta lo diferente, lo diverso, cristaliza en lo extraño, en lo raro, en lo inexplicable que es evidente parte de nosotros mismos.

\section{OBJETIVOS:}

Un objeto tan complejo morfológica, sintagmática y semánticamente como el automóvil, que mueve tal nivel de relaciones, en tantísimo planos, es, automáticamente, un misterio indescifrable para la antropología. En consecuencia desde una mirada más clásica de la antropología podría decirse que es un reto con el que los antropólogos podríamos sentirnos relativamente a gusto. $Y$ no porque el coche pueda ser entendido a un nivel comunitario $o$, en su defecto, como un estudio de caso, sino porque plantea en si gran parte de lo que una antropología clásica podría desear: plantea una holística de la realidad social, concentrando lo que creemos que somos y dándole forma material. Pero por otro lado en coche permite una teoría, cristaliza el significación del objeto para los sujetos, incluido el investigador, y una metodología, donde el objeto ilumina sobre el tiempo y el espacio que contextualiza al sujeto. Para la antropología más clásica sólo el ritual tenía la fuerza de materializar la teoría social, sin embargo el automóvil impone un ritual, en su conducción, en su compra, como objeto práctico y como deseo, y de está manera se convierte en un ritual del mundo contemporáneo. Esta teoría de lo social asociada al automóvil es un regreso al significado que puede, y de hecho lo hace, chocar con una metodología del automóvil, no ya sólo a un nivel de la disciplina antropológica, sino sobre todo de la social. La metodología no podría estar más en relación a un contexto social: el automóvil permite a sus usuarios, a las naciones y al simple espectador situarse él y la sociedad en unas determinadas coordenadas. Los anuncios en televisión(spots publicitarios) de estos objetos explotan este choque entre teoría y metodología de manera constante. Los coches de gama alta están cargados de más teoría y, consecuentemente, de significación y no necesitan demasiada metodología, por eso se les puede proponer en medio de parajes naturales que en nada contextualizan. Sin embargo, cuanto más baja es la gama más metodología implementan, lo que significa que el coche se diluye entre edificios y sistemas urbanos de ordenación del espacio.

\section{METODOLOGIA:}

No es nueva esta diferenciación radical entre metodología y teoría. En la antropología social que ha intentado por todos los medios hacerse ciencia había un acuerdo entre la metodología, el cómo hacerse con los datos, y la teoría, el cómo ordenarlos y, luego, ordenarlos. El 
problema, como no podría ser de otra manera, no proviene de ahí, sino de el por qué. En última instancia porque la metodología no es más que una teoría, un planteamiento de los significados en su aparataje histórico que aspira a tener un sentido cultural. El por qué es otra cosa. De las dos maneras que a priori podemos detectar, un por qué funcionalista y un por qué estructural, es evidente que la antropología tiende a la segunda aunque se ve tentada, por un simple ejercicio de cientificidad en sintonía con ciertas demandas sociales, a la primera. La resolución de la ecuación planteada entre el los cómo y los porqué tiene que darse en otra variante. En otras palabras la teoría tiene que ser una aspiración permanente frente a la voluntad de la acción. Evidentemente esto nos introduce en una serie de problemas que no son en absoluto fáciles de contestar, pero valga entender que estamos ante un ejercicio, el de la antropología, que tiene que plantear algún tipo de contestación y que esta no puede ser ni deducción ni, mucho menos, inducción de la acción. La antropología social sólo puede aspirar al pensamiento, a lo más conocimiento o, mejor dicho, al conocer, de forma experimental, si se quiere, pero nada más. En la falsedad de las dicotomías apriorísticas de las enseñanzas universitarias se etiqueta a los antropólogos entre los que se dedican a la acción, ya sea porque hacen "mucho" trabajo de campo, ya sea porque aplican lo que piensan, de los que se dedican a la teoría. Una falsedad como otra cualquiera. Todo es teoría en la antropología social, porque todo es teoría en la realidad social. Las formas de acción, como el ejercicio político, por ejemplo, se justifican y legitiman en la teoría. En última instancia porque el ser humano se piensa a si mismo, incluso cuando pretende sólo actuar.

Los que piensan en la acción tienen que tener una metodología, se dice, y poca teoría, acaso porque piensan en la transformación. Otra de esas tristes dicotomías: la teoría como fijación frente a la metodología como acción para la transformación. El criterio lamperuasino nos enseña que el cambio no significa transformación y que sólo el pensamiento está dotado de la fuerza para crear las condiciones del cambio. Incluso podríamos plantearnos que en un mundo tan dado a los cambios como es el occidental, que ha hecho de ello parte de su leitmotiv, cuáles son los lugares, los espacios, las verdaderas proporciones de la transformación. Si pensamos por un segundo en los automóviles como parte de este ejercicio no es difícil llegar a la conclusión que estamos ante un elemento que es acción, que es cambio: el coche se mueve, se conduce, cambia como objeto casi cada seis meses, transforma los espacios e, incluso, dicen algunos, la personalidad de los individuos. Objeto, aparentemente, hecho para y por la acción. Pero todo esto se disuelve en la reflexión: el objeto, el automóvil, es sólo una aspiración, de ahí su cambio permanente, hacía elementos menos permutables: el prestigio, la movilidad, el desarrollo de la propiedad privada, el mercado o la creencia de una cierta disciplina ciudadana. No se trata de valores pre-acción. Se trata de que la teoría es el núcleo duro de nuestra aspiración, como antropólogos, como ciudadanos, como sociedad.

BIBLIOGRAFIA:

Appadurai, Arjun (Edit.) (1991) La vida social de las cosas. Perspectiva cultural de las mercancías. México, Grijalbo, CONACULTA. (Orig. 1986).

Bourdieu, Pierre (1988) La distinción. Criterios y bases sociales del gusto. Madrid, Taurus. (Orig. 1979).

Flink, James J. (1990) TheAutomobileAge. Cambridge, MA, The MIT Press.

Geertz, Clifford (2005) Le souk de Sefrou. Sur l'économie de bazar. París, Bouchène. (Orig. 1979).

Jakle, John A.; Sculle, Keith A. (2005) Lots of Parking: Land Use in a Car Culture. Charlottesville, VA, University of Virginia Press.

Miller, Daniel (Edit.) (2001) Car Cultures. Materializing Culture. Oxford, Berg.

Urry, John (2003) Global Complexity. Cambridge, Polity.

Volti, Rudi (2006) Cars and Culture: TheLifeStory of a Technology. Baltimore, MD, Johns Hopkins UniversityPress.

Wollen, Peter; Kerr, Joe (2004) Autopia: Cars and Culture. Londres, Reaktion. 
De: José Luis Castillo <jlcastillo@ujaen.es>

Asunto: libro

Fecha: 15 de abril de 2013 20:51:16 GMT+02:00

Para: "Alejandro Jiménez Castro"<ajimenca@ucm.es>

Estimado Alex:

Me ha llegado tu libro, que me leído de una atacada, sintiendo una gran admiración de cómo tratas el tema y a la vez una enorme envidia por tu capacidad de trabajo y esa mirada profunda que te define, estoy contigo que la transición fue un performance social y esas entrevistas a los presos políticos demuestran que sigues apostando por la reflexión apoyada en datos empíricos. Me reitero, mi sincera enhorabuena. Aprovecho la ocasión para contarte que ya estoy metido en harina, mi preocupación por los automóviles empieza a mostrarme un camino (te acuerdas qué te lo comenté en la comida de la tesis de Murcia), me he hecho con la bibliografía básica y uno de mis doctorandos ha accedido ha trabajar sobre el tema; a la vez, que me he puesto encontacto con el grupo de sociólogos ingleses que tienen al automóvil como centro de su pensamiento, el propio Urry me anima a que haga mi aporte particular, lo que es mucho pedir, pero bueno hay estoy. Mi idea básica es intentar superar el nivel de que los automóviles son objetos del capital, lo que me llevaría a una descripción de los usuarios y sus implicaciones por las categorías clásicas de clase, edad y sexo; inclusopasar por encima el núcleo de funcionalismos clásicos de nuestra disciplina, intentando explicar la sociedad como si fuera un centro mecánico de estilo de vida, acaso comportamiento, siempre sujeto a "mentalidades". Ya sabes lo que me aburre todo esto. Todo es dado por hecho y una vez más olvidamos que no trabajamos para un organismo oficial, ni para unaempresa, que requiere "saber". En mis años de estudiante fui testigo de cómo se construía el consumidor, antes de tener ni el producto ni una sociedad que demandará nada de nada. Me acuerdo mucho de Santiago cuando llamaba aquellos estudios de corte cuantitativos las cantarinas, porque seguían la melodía que el director le marcaba... que pena lo de Santiago, me comentaron en el congreso de Salamanca, donde te echamos de menos, que lo despidieron de la editorial, a ver si un día lo llamo y me cuenta cómo le va. Bueno, como te decía, quería superar la idea de que el automóvil es un objeto más en relación con los parámetros sociales clásicos, entiendo que para aquellos que tienen el coche como centro de sus vidas comerciales e industriales los datos sean importantes, me hago cargo que para el Instituto de Estudios de Automoción (míratelo en la red, que no tiene desperdicio) esa sociología sea importante, pero a mi parecer todo esto puede describir una cierta situación social, pero ni la interpela ni la explica. Pero es que, además, me aburre toda esa manera de hacer unasciencias sociales que al final interesa sólo al que a hecho el encargo del estudio.Vasta que veas la manera en como se expresa la Dirección General de Tráfico de este país para observar que toda la realidad es reducida a una estadística que en el mismo saco mete elcomercio de los coches, los accidentes y las sanciones a los conductores...

Por lo tanto, quería entender el automóvilcomo algo más, por un lado, como un objeto tecnológicocontemporáneo que nos sirve de metáfora de la explicación de lo qué somos y dónde estamos y, por otro, como objeto que tiene una vida propia, tanto con un recorrido estético, industrial y comercial, cuanto más porque es el elemento clave de una compleja mirada de la democracia política vía empresarial, de los ideales de movilidad o de la capacidad de transformar desde nuestras economías domésticas hasta los paisajes, las ciudades y las formas de hacer sociedad.En efecto, no quiero caer en la idea exagerada de que los coches sean el centro explicativo de todo lo social en el mundo contemporáneo (ya me dieron de lo lindo cuando publique aquello del riesgo yno me apetece más), lo que me planteo es que hoy por hoy es uno de los "objetos" básicos para entender ese mismo mundo que ocupa de manera central. En cierta medida podemos decir, con ese anclaje en las ideas prístinas de Durkheim, que el tema es la relación que se establece entre las economías del capitalismo y la idea de que lo contemporáneo se centra en la estrecha relación con la movilidad y la intercomunicación, todo lo cual, es obvio que cristaliza en tres elementos, uno político, que es el mercado, otro simbólico, el automóvil, y, por ultimo, otrometafórico, que es el cyborg. Lo humano se reduce de muchas maneras diferentes a una serie de tecnologías que lo amplían, lo significan y lo identifican. En este sentido es en el que quiero pensar los automóviles, como objetos que se relacionan con sujetos en contextos de prácticas sociales determinadas. No se 
si todo esto te parece una de esas "tonterías" que pensamos desde la universidad o si tiene algo más de peso. En cualquier caso no quería caer una vez más en una idea simplista de lo qué es la sociedad, la sociología y las maneras críticas de pensarnos. Por todo ello te agradecería me comentaras tus ideas al respecto, estoy en ese momento que cualquier cosa es bienvenida. Por cierto me comenta Zacarías que ciertas sugerencias tuyas fueron claves para su proyecto de Cátedra. Es decir, que sigues siendo clave para nosotros y aquí estás siempre presente, a ver si montamos un seminario para el otoño y te vienes unos días con Cristina...¿ ¿Carlos sigue en San Francisco?Dile que si puede quede se quede allí, aquí la cosa está fatal y estos están descaradamente vendiendo todo a sus amigos, ni las migas nos van a dejar. Bueno no te entretengo más, un abrazo, JL

De: jlcastillo@ujaen.es

Para: Paco Ortega <webmaster@latortugadeulises.blogspot.com> Fecha: 16 de abril de 2013 23:12:05 GMT+02:00

Asunto: entrada blog

\section{EL AUTOMÓVIL ES UN SUEÑO MODERNO}

Cuando Jack Kerouac se suma a la Generación Beat, a mediados de los 50 del siglo XX, Estados Unidos esta saliendo de la crisis producida por la Segunda Guerra Mundial y la American Way of life está desarrollándose como nunca. Es el momento del automóvil, pasando de medio de transporte a símbolo de deseo de consumo y haciendo del conductor un consumidor. Una transformación que finiquitaba la idea de vivir simplemente como humanos y afirmando la idea de que somos nuestra tecnología. La Guerra había dejado una sensación de posibilidad y animaba a mostrarlo aplicando las tecnologías duales, de tanque a camión, de soldado a conductor, de ciudades con calles a la búsqueda de aparcamiento. En efecto, nada podía ser igual, la guerra había mostrado, por encima de cualquier otra cosa que el mundo era pequeño, domesticable y capitalista. $Y$ el símbolo de todo ello era el automóvil.La hipótesis ahora es la de la pura cibernética, en el siglo XX el imperio concentró el deseo sobre el coche y obviamente como objeto del presente se hacia permanentemente futuro. La democracia, como promesa política, se dio en el automóvil y su verdadera capacidad es la de involucrar todos los niveles de lo social. Incluso la rebeldía más absoluta sólo se podía dar como parte del discurso ambivalente de los automóviles.

De: “Alejandro Jiménez Castro" <ajimenca@ucm.es>

Asunto: Re: libro

Fecha: 18 de abril de 2013 08:28:16 GMT+02:00

Para: José Luis Castillo <jlcastillo@ujaen.es>

José Luis, Me alegra que te gustará el libro, aunque ya sabes que tu amigo Andrés Cortina me ha puesto a parir en el Babelia, creo que este tema de la transición está viciado y que a partir de ahora me voy a concentrar en escribir novelitas y algún ensayo para Anagrama, como hace él. Con respecto a lo que me comentas de tu trabajo ya sabes que en realidad poco o nada te puedo ayudar con el tema de los coches. Aunque te adjunto algunas ideas que me vienen al hilo de todo esto. Pero te reitero que poco te puedo ayudar.

La mirada de las ciencia sociales con un sesgoeconomicista (incluso te diría que marxista) encontró en los objetos más que simples cosas. Era todo un mundo de saberes, prácticas y de 
símbolos. Pero, además, la incorporación de categorías de análisis como ciudadanía o consumo estaban relacionadas más que con identidadessubjetivas con sujetos que se adscribían con practicas en relación a objetos. Entenderlos es, consecuentemente, clave para percibir las formas sociales y culturales de Occidente, y, por qué no, a todo lo que tiene de occidental el resto del mundo. En este sentido puede decirse que los objetos tomaban una suerte de hermenéutica del capitalismo, donde no hay interpretación sin establecer niveles y no hay niveles sin procesos simbólicos. Estos niveles (simbólicos) están en relación con ciertos criterios de consumo, concentrado en la moda como soporte estético, los automóviles, los objetos del hogar, cuando no el propia arquitectura en si misma y las formas de ocio; en ultima instancia significados que muestran la quintaesencia de la sociedades del espectáculo capitalista. Pero si los objetos significaban verdades, es decir, eran capaces de dar contenido a los individuos y formaban representaciones sociales, también revelaban la capacidad para recrear funcionalidades. La ropa o los automóviles tienen la capacidad crear representaciones donde es muy complejo establecer la frontera entre el para qué sirven, acaso ya no para vestirnos o transportarnos sino somos símbolos de practicas que rearman los conceptos relacionados con el gusto (el deseo), la clase, la posición, él género, la edad e, incluso, los criterios étnicos.

Los objetos son, además, elementos que tienen que ver con el consumo y con el deseo o, dicho de otra manera, los objetos devienen en consumo vía deseo. El simple deseo es consumo, lo que, en cierta medida, viene a significar que es un fetiche y pierde así su capacidad de asumir una mirada única sobre su fabricación, transformación y distribución. Los planteamientos más clásicos de la antropología económica ponían el acento en la cadena producción-distribución-consumo, pero desde hace años esto no es válido para los objetos capitalistas, relacionados sólo con el consumo. Hasta el punto de que la produccióndistribución es, o también consumo, o parte de realidades relacionadas con elementos de economía subalterna y emergente. Es lo que Pierre Bourdieu llama la potencia mercantil de los objetos, es decir, que para que un objeto sea socialmente relevante tiene que ser característicamente mercantil. El objeto es en la sociedad capitalista un elemento de consumo relacionado con su potencialidad mercantil. Para estas miradas los objetos tiene una suerte de vista social y pasan por lo que Appadurai entiende como las fases de transición, lo que viene a ser los diferentes contextos por los que los objetos pasan a lo largo de su existencia. Obviamente una existencia siempre social, lo que les deifica y simboliza de manera diferente según la fase. Los objetos que produce-consumen las sociedades capitalistas pasan por una serie de fases que incluyen la fase en que están en producción, en distribución o en otras de sus fases mercantiles. Incluso como parte del uso tiene un valor mercantil.

En fin que no quiero que traigas el tema a donde no quieres, sólo que tengas en cuenta nuestramirada, ya olvidada, del marxismo como respuesta.

Lo del seminario en tu universidad de momento imposible, tengo la agenda que muero, de hecho, queremos ir a California este verano a ver al niño y no se si después de Santander me podré cuadrar con Cristina. Un abrazote.

De: José Luis Castillo <jlcastillo@ujaen.es>

Asunto: Propuesta de comunicación

Fecha: 18 de abril de 2013 13:17:40 GMT+02:00

Para: "Secretaria congreso" <secrataria@ceidal.pt>

Les adjunto el título y resumen de mi comunicación para el simposio 14, les agradecería me confirmaran la recepción.

Autores: José Luis Castillo Fernández Félez (Universidad e Jaén); Antonio Malpica Pons (Universidad Rey Juan Carlos. Madrid). 
Titulo: LOS OBJETOS DEL CAPITALISMO, ENTRE LO SOCIAL Y LO INDIVIDUAL.

Resumen: La estrecha relación de automóvil y vivienda se establece en estos juegos tensionados entre lo individual y lo social. El automóvil, tan individual, ha terminado por modelar el urbanismo, tan social, en acuerdos políticos que, sin duda, son muy extremos en aquellos sitios donde la cultur car ha triunfado sin limitaciones. En México, donde el transporte público sigue siendo una realidad en la mayoría de sus ciudades tener un coche tiene un significado e lo individual y lo social muy diferente de aquellos sitios donde, como en Los Ángeles, prácticamente se ha reducido a su mínima expresión, o con referencia a Europa, donde el aparcamiento es tan caro como difícil de encontrar. El automóvil tiene connotaciones políticas según se plantee un uso y distribución de lo individual y lo social. Obviamente implica un grado mayor de individualidad apostar por el automóvil que por el transporte público, pero no significa que rompa la tirantez en la arena política, pues más automóviles también implica más carreteras, aparcamiento y facilidades en la compra o los seguros, a la vez que más normatividad, política, control y mayor riesgo de colapso acústico, ambiental y urbanístico. Como representación política el automóvil está en el centro de todas estas tensiones, hasta el punto que todo lo que significa como elementos de libertad y amplificación de individuo es, a la vez, un mayor grado de normatividad y significación de la vida en sociedad.

De: "cortina, andres" <cotinagom@uniovi.es>

Asunto: Re: Babelia

Fecha: 22 de abril de 2013 15:02:12 GMT+02:00

Para: José Luis Castillo <jlcastillo@ujaen.es>

José Luis, la verdad que el viaje desde Baeza fue muy agradable y la charla contigo muy instructiva. De tu comentario he deducido algunas cosas y no entiendo el cabreo de Jiménez Castro, en mi reseña para EL PAIS le salvo la cara y el libro,que en realidad es muy malo y no aporta nada; de hecho, obvia que han pasado más de treinta años de excelentes trabajos sobre el tema. Y sino fuera porque es un histórico no pasaría de ser un alumno aventajado. Además ahora resulta que va de padre de todos. Por cierto, si vas con el corre-ve-y-dile le recuerdas que yo sigo viviendo donde siempre y que no mande mensajitos al departamento haciéndose de nuevas. Saluda a Elena, Andrés

De: José Luis Castillo <jlcastillo@ujaen.es>

Asunto: Re: Re: libro

Fecha: 23 de abril de 2013 00:15:57 GMT+02:00

Para: "Alejandro Jiménez Castro"<ajimenca@ucm.es>

Alex, Me gusta esa idea que aportas sobre superar el simple nivel del objeto e incluirlo en una perspectiva más de fetichismo. Desde mi punto de vista el tema es complejo y no quería caer en decir un montón de tópicos; de hecho, una de las primeras tentaciones que hay que superar, y te puedo afirmar que no es fácil, es tomar a los automóviles como objetos etnográficos per se (como si fueran sólo núcleos de una estética a lo Gadamer). Y no se trata sólo de obviar el sentido cuasi semiótico o el análisis del objeto como representado, sino intentar preñarlo de algún análisis social. Pero no es fácil, ya te digo, simplemente porque el automóvil está ahí, se hace presente por encima de casi cualquier otra consideración, tomando un papel protagonista que no permite ver más allá. Ya no es sólo que el propio investigador utilice un coche, sino que prácticamente se mire donde se mire están los coches y en nuestra 
sociedad prácticamente no hay lugar que el coche no sea una parte definitoria del paisaje. En consecuencia, tanto nivel de información es difícil de tratar. Además, el automóvil por definición no se está quieto, es un objeto que se usa y la movilidad le define (aunque creo que habría que decir que le contiene más que le identifica). Pero esto me lo tengo que pensar poco a poco, lo que ahora me gustaría resaltar es que nos enfrentamos a un objeto de estudio multiplicado (creo que en estote sigo en lo que dices en tu mail), pero escurridizo, amplificado e hibridizado. Pero en fin sigo pensándome todo esto. Por cierto me encontré a Andrés Cortina en un acto de la UNIA y te manda saludos, sigue como siempre. JL

De: “BERNARDO 003" <bernadoesperso0042@hotmail.com>

Asunto: Re: Re: Re: horario tutoría

Fecha: 23 de abril de 2013 12:20:11 GMT+02:00

Para: José Luis Castillo <jlcastillo@ujaen.es>

Profesor, ya me he leído el libro del mercado que me dijoy piensa lo mismo k yo, pr el tfnon tengo claro si tengo primero que poner la metodología o primero también la otrateoría. Me dijo que la introducción lo escribiera lo ultimo, pero después de k. Además aviso de que el grupo de gente $k$ estudiado me dicen $k$ ellos no saben nada de comprar y vender coches, por lo que creo que voy a entrevistar a uno de ellos que ha tuneado muy guapo el coche de su madre. Lo que me tiene que decir también y si le hago una historia de vida como hicimos para su asignatura y si hay algún guion diferente y que me pueda bajar de internet. Hasta el martes en la tutoría

De: webmaster@latortugadeulises.blogspot.com

Asunto: Re: comentario RE: El automóvil es un sueño moderno

Fecha: 23 de abril de 2013 11:22:31 GMT+02:00

Para: CASTILLO JL jlcastillo@ujaen.es

Nos llega está entrada al blog para que apruebes, obviamente, que has despertado la caja de los truenos con lo de los coches. Por cierto para la otra semana mándame alguna imagen con la entrada. ¿A este tipo lo vas a contestar ahora o antes lo apruebas? Tu me dices.

Inicio del mensaje reenviado:

De: acordinadero@gmail.com

Para: replies@latortugadeulises.blogspot.com>

Fecha: 20 de abril de 2013 14:37:46 GMT-04:00

Asunto: comentario RE: El automóvil es un sueño moderno

No es que no este acuerdo, al contrario, lo que creo es que hay otra mirada. En Puerto Rico estamos estudiando los sistemasdisciplinares de Guattari como dispositivos y tenemos una visión menos desde el imperio. De Hecho,"La historia del automóvil"(Citroën 10 H.P.),de llyaEhrenburg y publicado en 1925, viene a demostrar que no es sólo Estados Unidos el lugar donde el carro se da con una fuerza desmedida, hasta ser la parte explicativa de su fisonomía como País, sino que Europa y concretamente Francia representan el modelo alternativo. Parece que todo se reduce a una misma "verdad": el automóvil es el lugar privilegiado de la representación de la modernidad. Ehrenburg es más conocido por su eterno disentir como disidente político soviético, dejando una actitud muy de critico de la crítica hacia el mundo del siglo XX, aunque sin olvidar su mirada marxista y su ingenuidad con sus referentes. Pero 
dentro de toda su obra es en ese libro menoren donde nos muestra, quizás, que la relación con la tecnología, con la fe ciega en el desarrollo industrial, en las posibilidades de la movilidad, en la seguridad es un espejismo que no tiene nada de natural. En la entrada del doctorCastillo se centra en la idea de que todo se reduce a la movilidad, pero dando cuenta de autores como Ehrenburg descubrimos que hay también una zona oscura en torno al mundo del carro y que estárepresentado en la fábrica, el accidente o el endeudamiento.

De: "Grupo de Investigaciones en Movilidad" <GIM@googlegroups.com>

Asunto: Newsletter - April 2013

Fecha: 23 de abril de 2013 16:35:15 GMT+01:00

Para: undisclosed-recipients:

Los automóviles como objetos característicos del mundo capitalista devienen en objetos mercantiles no solo, que también, por mercancías, sino que lo son también como objetos del capital que muestran el mercantilismo del símbolo, de su posesión y de los sentimientos que generan. Es decir, que fuera de ser elementos funcionales con lógicas de economía racionalista son, además, objetos que mecanizan la capacidad de desearlos y poseerlos. Los automóviles, en este sentido, hablan de los dueños y éstos hablan vía sus automóviles. Una suerte de comunicación social con un complejo código que se relaciona con elementos de clase, género, edad e, incluso, etnia. Consecuentemente, el automóvil, como objeto, es inminentemente social y expresa en sus consideraciones básicas las cosas que los individuos piensan de su papel en la sociedad, lo que implica no sólo códigos sociales asumidos, sino, también, proyectados. Pero el automóvil como objeto social tiene, cuando menos, otros dos elementos más en su propia hermenéutica: su capacidad de movilidad y transporte, lo que tiene añadidos de significados, las transformaciones urbanas o la pérdida/ganancia de movilidad de los sistemas colectivos y, por otro lado, el automóvil como parte de un entramado industrial, lo que va desde pautas empresariales a las asumidas nacionalidades de los objetos propios, más a más con un elemento que nació a la par que las grandes teorías nacionalistas y que se asumió como retos puramente nacionalistas.

Dicho esto podemos observar que los automóviles tienen tres niveles de análisis mínimos: uno, simbólico-mercantil, otro, socio-industrial y, por último, una evocación del ordenamiento social. El problema ahora es que si bien estas coordenadas tendrá que ser los elementos rectores durante el trabajo de campo, la cuestión tiende a diluirse por otros muchos motivos y que, en líneas generales, son compartidos por cualquier trabajo de campo que se quiera abordar. En este sentido, la toma de posición durante el trabajo, la lejanía, la búsqueda de informantes y la resolución de los problemas básicos de la vida cotidiana son los mismos. El problema se inscribe, consecuentemente, en otro orden de cosas, uno la, digamos, extraña relación entre los automóviles y sus dueños y la delimitación, más o menos precisa de cual es exactamente el lugar que ocupa en lo soical, y que sirve para entender, también, cuál es ese otro entramado de gustos, necesidades y deseos que terminan por normalizar ciertos objetos de donde el automóvil es clave.

De: "Maria Asunción Calleja" <macalle@ujaen.es>

Asunto: [de3714] Dirdep

Fecha: 24 de abril de 2013 09:17:05 GMT+02:00

Para: dep3714@ujaen.es 
Estimados compañeros adjunto os envío un texto que puede servir de base para abordar el punto 3 del inmediato Consejo de Departamento (previsto para el 29):

3. Atención, si procede, a la petición por la Asamblea de Trabajadores de la UJA de debate y valoración del Informe de la Comisión de Expertos del Ministerio.

Se repartirán copias en el Consejo.

De la misma manera queda pendiente que al punto 7 del consejo traigáis la petición de los seminarios que habíamos aprobado en el consejo extraordinario anterior:

Antropología: Vivir entre maquinas, del automóvil al cyborg.

Historia Moderna: Iniciación a la paleografía.

Geografía Física: Refuerzo a la asignatura del Grado.

Un saludo

De: "Antonio Malpica"<amalpica@urjc.es>

Asunto: Re: Congreso portugal

Fecha: 23 de abril de 2013 08:40:07 GMT+02:00

Para: JLCASTILLO<jlcastillo@ujaen.es>

José, tenemos que hablar de unas cosas para el congreso del CEIDAL, incluido el alojamiento, luego me cuentas. Por cierto, me dice Maricarmen que no tiene la dirección de los de alicante, que se la mandes. Bueno que siento meterte en estos líos de la aneca pero es que ahora soy también el secretario de todos. Dime que te parece esto. Llámame el viernes por la mañana que el finde me voy de boda a Béjar.

De: José Luis Castillo <jlcastillo@ujaen.es>

Asunto: Re: Proyecto tesis

Fecha: 24 de abril de 2013 23:37:36 GMT+02:00

Para: "PEÑALANDAJUANMANUEL"<jmpenal@autlook.com>

Juan Manuel, no te olvides que está tesis está dentro de un proyecto de investigación del departamento por lo que tienes que incluir algunas referencias de otro tipo. Por lo demás decirte que tu pregunta de porque investigamos esto y cual es mi mirada del automóvil tiene también parámetrospersonales (y me gustaría que vieras como parte "modelo" para tu tesis), en última instancia tienes que encontrar tu estilo y tus razones. Veras que te adjunto unas lecturas, mira a ver que se puede conseguir-interesar, he preparado una carpeta con artículos y con otro vaciado que le metas al ISI creo que estarás más o menos listo. Nos vemos el martes por la tarde, es que ahora ando muy liado y no puedo quedar para antes. JL

David M. Haugen, Matthew J. Box (Editor) (2005) Examining Pop Culture. Cars. GreenhavenPressISBN: 0737725435

Daniel Miller (2006) Starting a Small Restaurant. Harvard CommonPressISBN: 1558322876 
Heather Horst, Daniel Miller (2006) TheCellPhone: AnAnthropology of Communication. BergPublishersISBN: 1845204018

Fred R. Myers (Editor) (2001) TheEmpire of Things: Regimes of Value and Material Culture. School of American ResearchPress ISBN: 1930618069

FrancesBasham, Bob Ughetti, Paul Rambali (1994) Car Culture. Plexus Publishing ISBN: 0859650324

Gerald Raunig (2008) Mil máquinas. Breve filosofía de las máquinas como movimiento social. Traficantes de SueñosISBN: 978-84-96453-33-2

De: José Luis Castillo <jlcastillo@ujaen.es>

Asunto: Re: Re. Congreso portugal

Fecha: 25 de abril de 2013 21:09:58 GMT+02:00

Para: "Malpica Gómez, Antonio"<amalpica@urjc.es>

OK. Como veo lo que me mandas se me ocurre otra cosa. Perdona se me ha olvidado decirte que ya tengo las cifras, si quieres te las mando, aunque ahora es mejor dejarlo en algo más especulativo, siguiendo tus ideas. Pero ya veremos, porque lo de Portugal ya lo mandé, el resumen, y quizás mejor guardarnos esto para otra cosa, pensaba si no un artículo para la revista de Jorge. Mañana te llamo.

Por cierto, te tengo que mandar un texto con lo que hablamos en el congreso de Manchester, es que por ahora no le veo mucho sentido. Bueno que no te entretengo que el viernes te digo.

De: "Alejandro Jiménez Castro" <ajimenca@ucm.es>

Asunto: $R e: R e: R e:$ libro

Fecha: 26 de abril de 2013 06:34:17 GMT+02:00

Para: José Luis Castillo <jlcastillo@ujaen.es>

Castillo, si en algún momento ves a Andrés le dices muy amablemente que yo me paso lo que diga el Babelia por ahí, que escribí en El País durante años cuando tocaba de verdad, antes de los 80. Y que si quiere dar clases de ortodoxia marxista que se apunte con sus amigos de Toulouse al congreso de la ESA. Saludos. 